\title{
Relationship between subtle urinary albumin excretion and risk of incident hypertension: modification by glomerular filtration rate
}

\begin{abstract}
Masanori Munakata, Tomomi Hattori and Satoshi Konno
It has been reported that an increase in urinary albumin excretion (UAE) within the normal range could be a risk factor for incident hypertension. However, it remains unclear how the subtle increases in UAE and renal function interact in the development of hypertension. We examined the modification of UAE as a risk factor for incident hypertension by glomerular filtration rate (GFR) in the Japanese population. We prospectively followed 1281 normotensive individuals from Watari town (34.3\% men; mean age, $58.0 \pm 12.3$ years old) whose UAE was $<30 \mathrm{mg} \mathrm{g}^{-1}$. Cr. Hypertension was diagnosed as a systolic blood pressure (BP) $\geqslant 140 \mathrm{~mm} \mathrm{Hg}$ and/or a diastolic $B P \geqslant 90 \mathrm{~mm} \mathrm{Hg}$, or antihypertensive medication use. The relationship between sex-specific quartiles of UAE and incident hypertension was examined with Cox proportional hazard analysis. During a mean follow-up of 3.7 years, 315 individuals developed hypertension. Multivariate Cox proportional hazard analysis revealed that a subtle increase in UAE was a risk factor for incident hypertension, but there was a significant interaction between UAE and estimated GFR (eGFR) $(P=0.018)$. The risk of incident hypertension dose dependently increased in the highest eGFR quartile $\left(\geqslant 90 \mathrm{ml} \mathrm{min}-1\right.$ per $1.73 \mathrm{~m}^{2}$ ). Decline in renal function alone increased the risk of incident hypertension but the increased risk with a subtle increase in UAE became smaller and less clear in the lower eGFR quartiles. The present data suggest that UAE as a risk factor for incident hypertension is largely dependent on eGFR levels.
\end{abstract}

Hypertension Research (2017) 40, 994-998; doi:10.1038/hr.2017.77; published online 21 September 2017

Keywords: glomerular filtration rate; urinary albumin excretion

\section{INTRODUCTION}

High blood pressure (BP) is the most significant risk factor for cardiovascular and cerebrovascular diseases in the world. ${ }^{1}$ The prevention of high $\mathrm{BP}$ is becoming increasingly important to reduce medical costs in many countries. The kidney has a central role in the regulation of long-term BP homeostasis ${ }^{2}$ and this is accomplished via volume control through renal manipulation of sodium excretion. In other words, impairment of sodium excretion could lead to long-term BP elevation.

Urinary albumin excretion (UAE) is a well-known marker of renal endothelial damage. A series of human studies showed that not only microalbuminuria, but also significantly lower levels of UAE are associated with increased risk of incident hypertension or increased $\mathrm{BP}$ in the normotensive population, ${ }^{3-8}$ although there is one exception. ${ }^{9}$ These data strongly suggest that glomerular endothelial damage precedes the onset of hypertension, although the etiology of the subtle UAE remains unclear. On the one hand, the reduction in glomerular filtration surface area, which could be reflected by a mildly reduced glomerular filtration rate (GFR), may be a risk factor for the development of hypertension as a result of a limited ability to excrete sodium. This hypothesis is supported by human autopsy evidence indicating that young hypertensive individuals have a reduced number of nephrons compared with age-matched normotensive controls. ${ }^{10}$ It could be hypothesized that mild renal endothelial damage and renal function would interact in the course of hypertension development. Only the Prevention of Renal and Vascular End-stage Disease (PREVEND) study reported a significant interaction between UAE and GFR. ${ }^{4}$

The etiology of essential hypertension is markedly different between Caucasians and East Asians in terms of genetics and lifestyle. In this regard, the interaction between UAE and GFR may be different between them, because salt intake and its sensitivity is higher in the East Asian population. ${ }^{11}$ The Watari study is a prospective cohort study that began in 2009 to examine the prognostic significance of albuminuria for the prediction of cardiovascular events. ${ }^{12-14}$ The aim of this study was to use this population to examine whether or not a subtle increase in UAE within the normal range could be a risk factor for incident hypertension and if so, to determine how the risk is modified according to estimated GFR (eGFR).

\section{METHODS}

Study design and population

The Watari study is a prospective cohort study of the Japanese general population that was conducted in the town of Watari, Miyagi Prefecture, 
Japan. The study was started in 2009 in association with annual specific health check-up initiated by the government in 2008. The aim of this study was to examine the ability of albuminuria to predict cardiovascular events in the Japanese population. A detailed description of the study has been published elsewhere. ${ }^{12-14}$ The study protocol was approved by the ethics committee of Tohoku Rosai Hospital. Our study conformed to the Declaration of Helsinki and written informed consent was obtained from all participants. Participants included individuals from Watari (age, 30-79 years, $42.6 \%$ male), who received an annual medical check-up in $2009(n=3628)$.

\section{Measurements}

Information regarding past and current medical history and lifestyle factors (that is, smoking status, regular exercise and the amount of alcohol intake per drinking day) were examined using a standardized questionnaire specified for the health check-up. ${ }^{15}$ People who answered 'yes' to the question 'Are you a current regular smoker?' were categorized as 'current smokers' and people who answered 'yes' to the question 'Have you been exercising at least 2 days per week, at least $30 \mathrm{~min}$ each at an intensity that causes a slight sweat, for at least 1 year?' were categorized as doing 'regular exercise.' Being a 'heavy drinker' was defined as an individual who drinks $\geqslant 2$ gou (gou = Japanese sake unit; 1 gou contains $\sim 23 \mathrm{~g}$ of ethanol) per day for men and $\geqslant 1$ gou per day for women. ${ }^{15}$ Height, body weight and waist circumference were measured by trained nurses. Body mass index (BMI) was calculated as weight $(\mathrm{kg})$ divided by height $(\mathrm{m})$ squared. After 5 min of sitting, BP was measured using a semiautomatic cuffoscillometric sphygmomanometer (BX-10; Omron Colin, Kyoto, Japan). BP was measured two times if the first measurement was $\geqslant 140 \mathrm{~mm} \mathrm{Hg}$ for systolic or $\geqslant 90 \mathrm{~mm} \mathrm{Hg}$ for diastolic and the mean values were taken as representative $\mathrm{BP}$ values. If first measurements were $\leqslant 140 / 90 \mathrm{~mm} \mathrm{Hg}$, the single measurement values were taken as representative BP values. Blood samples were collected after an overnight fast and then analysed for low-density lipoprotein and high-density lipoprotein, cholesterol, triglycerides, HbAlc National Glycohemoglobin Standardization Program (NGSP), uric acid, and creatinine levels. HbAlc level was determined using high-performance liquid chromatography (HLC723G7; Tosoh, Yamaguchi, Japan) and the levels of the remaining biochemical markers were determined using a standard automatic analyser (7700DD; Hitachi, Tokyo, Japan). ${ }^{12-14}$ eGFR was calculated using the formula provided by the Japanese Society of Nephrology: eGFR $\left(\mathrm{ml} \mathrm{min}^{-1}\right.$ per $\left.1.73 \mathrm{~m}^{2}\right)=194 \times$ creatinine $\mathrm{e}^{-1.094} \times$ age $^{-0.287}(\times 0.739$ if female $) .{ }^{13}$ Morning spot urine samples were also collected for the measurement of UAE. ${ }^{12-14}$ Urinary albumin and creatinine concentrations were determined using the turbidimetric method (Au600; Olympus, Tokyo, Japan) and enzymatic method, respectively. UAE was expressed as the urine albumin-to-creatinine ratio. BPs were measured annually by the same protocol during the next 5 years on the occasion of governmental specific health check up.

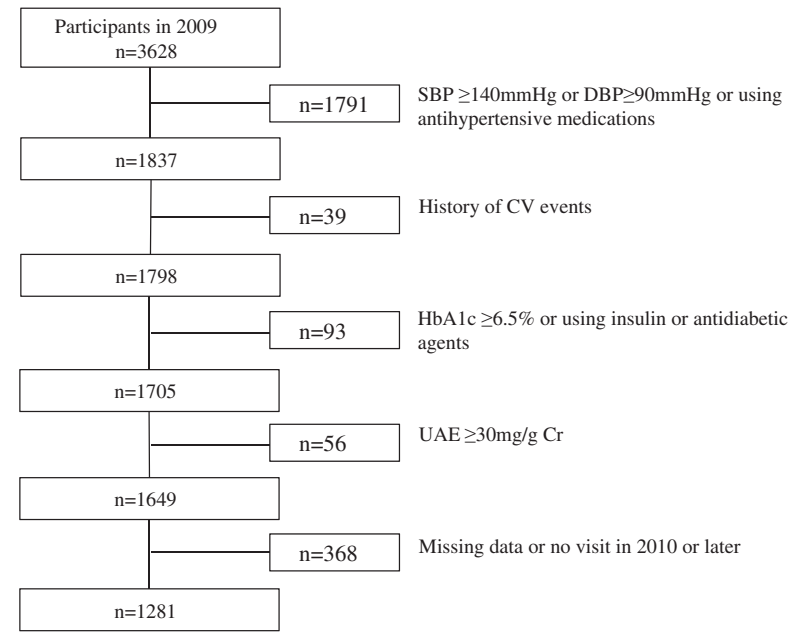

Figure 1 Flow chart of the study participants.

\section{Statistical analysis}

Of 3628 participants, 2437 were excluded from the analysis because of the following reasons: prevalent hypertension (systolic BP $\geqslant 140 \mathrm{~mm} \mathrm{Hg}$, diastolic BP $\geqslant 90 \mathrm{~mm} \mathrm{Hg}$ or the use of antihypertensive medications; $n=1791$ ), history of cardiovascular or cerebrovascular events $(n=39)$, diabetes mellitus (HbAlc $\geqslant 6.5 \%$ or use of insulin or antidiabetic agents; $n=93$ ), UAE $30 \mathrm{mg} \mathrm{g}^{-1}$. Cr or higher $(n=56)$, missing data $(n=167)$, or no visit in 2010 or later $(n=201)$. The remaining 1281 were included for analysis (Figure 1). The follow-up rate for the next 5 years was $89.2 \%, 74.5 \%, 71.3 \%, 67.8 \%$ and $66.2 \%$, respectively. We examined the relationship between UAE and incident hypertension on follow-up. Hypertension was defined as systolic BP $\geqslant 140 \mathrm{~mm} \mathrm{Hg}$, diastolic BP $\geqslant 90 \mathrm{~mm} \mathrm{Hg}$ or the initiation of antihypertensive medications. Participants were censored after being diagnosed as hypertension. Data are presented as mean \pm s.d., median (interquartile range) or percentage. Group comparisons were performed using the $t$-test, analysis of variance or a $\chi^{2}$-test. We used the Cox proportional hazards model to examine the correlation between UAE and incident hypertension. UAE was treated as a categorical variable. In the categorical analyses, we used sexspecific quartiles of UAE within the normal range, considering sex-related differences in urine creatinine excretion. ${ }^{3}$ Models were adjusted for the following covariates, which are usually viewed as related to the incidence of hypertension: age, sex, BMI, systolic and diastolic BPs, triglyceride, low-density lipoprotein, HbAlc, uric acid, eGFR, smoking, exercise habits and heavy drinking. The final multivariate model was tested for the interaction between UAE and eGFR. Similar analysis was done also by logistic regression analysis.

For subsequent analyses, we examined the incidence of hypertension by quartiles of UAE and eGFR ( $\geqslant 90,77-89,72-76$ and $<72 \mathrm{ml} \mathrm{min}^{-1}$ per $1.73 \mathrm{~m}^{2}$ ). All statistical analyses were performed using IBM SPSS statistics 20 for Windows (IBM Corp., Armonk, NY, USA). A $P$-value $<0.05$ was considered to be statistically significant.

\section{RESULTS}

Table 1 shows the baseline characteristics by UAE quartiles. Quartile ranges for UAE in men were 1.2 to $4.6,4.7$ to $5.9,6.0$ to 8.3 and 8.4 to $29.9 \mathrm{mg} \mathrm{g}^{-1}$. Cr, respectively, and those in women were 1.2 to $5.6,5.7$ to $7.7,7.8$ to 10.3 and 10.4 to $29.4 \mathrm{mg} \mathrm{g}^{-1}$. Cr, respectively. Age, systolic and diastolic BPs, triglyceride level and low-density lipoprotein level dose dependently increased with an increase in the UAE quartiles, whereas sex, high-density lipoprotein and uric acid concentration did not differ significantly among the four groups. Waist circumference was significantly increased with an increase in UAE quartiles although changes in BMI did not reach a statistically significant level. Distribution of eGFR categories significantly differed among four UAE quartiles, that is, high prevalence of eGFR Quartile 1 and low prevalence of eGFR Quartile 4 in the highest UAE quartile and vice versa in the lowest UAE quartile.

During a mean follow-up period of 3.7 years, 315 participants $(24.6 \%)$ developed hypertension. Compared with persistently normotensive subjects, participants who developed hypertension were older, more likely to be male and had higher BMI, and systolic and diastolic BPs. Moreover, both lipid and glucose profiles were worse in participants with incident hypertension. UAE was slightly higher and eGFR was slightly lower in participants with incident hypertension than in those with persistent normotension (Supplementary Table 1). The incidence of hypertension increased with an increase in UAE quartiles $(14.7 \%, 24.7 \%, 26.0 \%$ and $32.7 \%$, respectively, $P<0.001)$.

Table 2 shows the results of the Cox regression analysis. In the unadjusted model, the hazard ratios for incident hypertension of the second, third and fourth UAE quartiles in comparison with the first quartile were 1.73 (95\% confidence interval: 1.20-2.48), 1.77 (1.24$2.54)$ and 2.35 (1.66-3.33), respectively. After adjustment for known covariates (model 2) or lifestyle factors (model 3), the dose-dependent associations were slightly altered as indicated by the loss of significance of the third quartile group relationships. Those of the second and fourth quartiles remained significant. The associations, however, were 
Table 1 Baseline characteristics by quartiles of urinary albumin excretion

\begin{tabular}{|c|c|c|c|c|c|c|}
\hline \multirow[b]{2}{*}{ Variables } & \multirow[b]{2}{*}{ Overall $(n=1281)$} & \multicolumn{4}{|c|}{$U A E$} & \multirow[b]{2}{*}{ P-values } \\
\hline & & Quartile 1 & Quartile 2 & Quartile 3 & Quartile 4 & \\
\hline Men (\%) & 34.3 & 33.9 & 34.6 & 34.6 & 34.3 & 0.997 \\
\hline Body mass index $\left(\mathrm{kg} \mathrm{m}^{-2}\right)$ & $22.5 \pm 3.0$ & $22.1 \pm 2.9$ & $22.5 \pm 3.2$ & $22.6 \pm 2.9$ & $22.7 \pm 3.1$ & 0.057 \\
\hline Waist circumference $(\mathrm{cm})$ & $82.1 \pm 8.8$ & $80.6 \pm 8.9$ & $82.2 \pm 9.0$ & $82.4 \pm 8.3$ & $83.0 \pm 8.8$ & 0.005 \\
\hline $\mathrm{HDL}\left(\mathrm{mg} \mathrm{dl}^{-1}\right)$ & $65.3 \pm 15.9$ & $66.5 \pm 14.9$ & $65.3 \pm 15.4$ & $64.4 \pm 16.5$ & $65.0 \pm 16.7$ & 0.396 \\
\hline $\operatorname{LDL}\left(\mathrm{mg} \mathrm{dl}^{-1}\right)$ & $123.8 \pm 30.5$ & $118.8 \pm 29.9$ & $124.1 \pm 32.0$ & $126.0 \pm 29.7$ & $126.2 \pm 29.7$ & 0.007 \\
\hline $\mathrm{HbAlc}(\%)$ & $5.8 \pm 0.3$ & $5.6 \pm 0.3$ & $5.8 \pm 0.3$ & $5.8 \pm 0.3$ & $5.8 \pm 0.3$ & $<0.001$ \\
\hline Uric acid $\left(\mathrm{mg} \mathrm{dl}^{-1}\right)$ & $4.7 \pm 1.2$ & $4.8 \pm 1.2$ & $4.7 \pm 1.1$ & $4.7 \pm 1.1$ & $4.6 \pm 1.2$ & 0.455 \\
\hline eGFR $\left(\mathrm{ml} \mathrm{min}-1\right.$ per $\left.1.73 \mathrm{~m}^{2}\right)$ & $80.3 \pm 15.0$ & $78.6 \pm 13.9$ & $80.4 \pm 15.7$ & $80.9 \pm 14.5$ & $81.3 \pm 15.5$ & 0.096 \\
\hline UAE (mg g $\left.{ }^{-1} \cdot \mathrm{Cr}\right)$ & $7.0(5.2,9.8)$ & $4.2(3.6,4.9)$ & $6.1(5.6,6.9)$ & $8.2(7.3,9.0)$ & $13.1(11.1,16.8)$ & $<0.001$ \\
\hline Dyslipidemia treatment (\%) & 8.6 & 6.1 & 8.4 & 10.2 & 9.7 & 0.246 \\
\hline Smoking (\%) & 15.5 & 15.3 & 16.6 & 15.9 & 14.3 & 0.882 \\
\hline Exercise habit (\%) & 36.0 & 30.4 & 34.3 & 37.5 & 41.7 & 0.022 \\
\hline Heavy drinking (\%) & 4.8 & 5.1 & 4.8 & 5.1 & 4.4 & 0.969 \\
\hline
\end{tabular}

Abbreviations: BP, blood pressure; eGFR, estimated glomerular filtration rate; HDL, high-density lipoprotein cholesterol; LDL, low-density lipoprotein cholesterol; UAE, urinary albumin excretion. Data are expressed as mean \pm s.d. or median (interquartile range) for continuous variables and as percentage for categorical variables.

eGFR Quartiles 1, 2, 3 and 4 indicate 90-169, 77-89, 72-76 and 40-71 $\mathrm{ml} \mathrm{min}^{-1}$ per $1.73 \mathrm{~m}^{2}$, respectively.

$P$-values indicate the results by analysis of variance or chi square test.

The bold values indicate statistically significant components.

Table 2 Results of Cox regression analysis

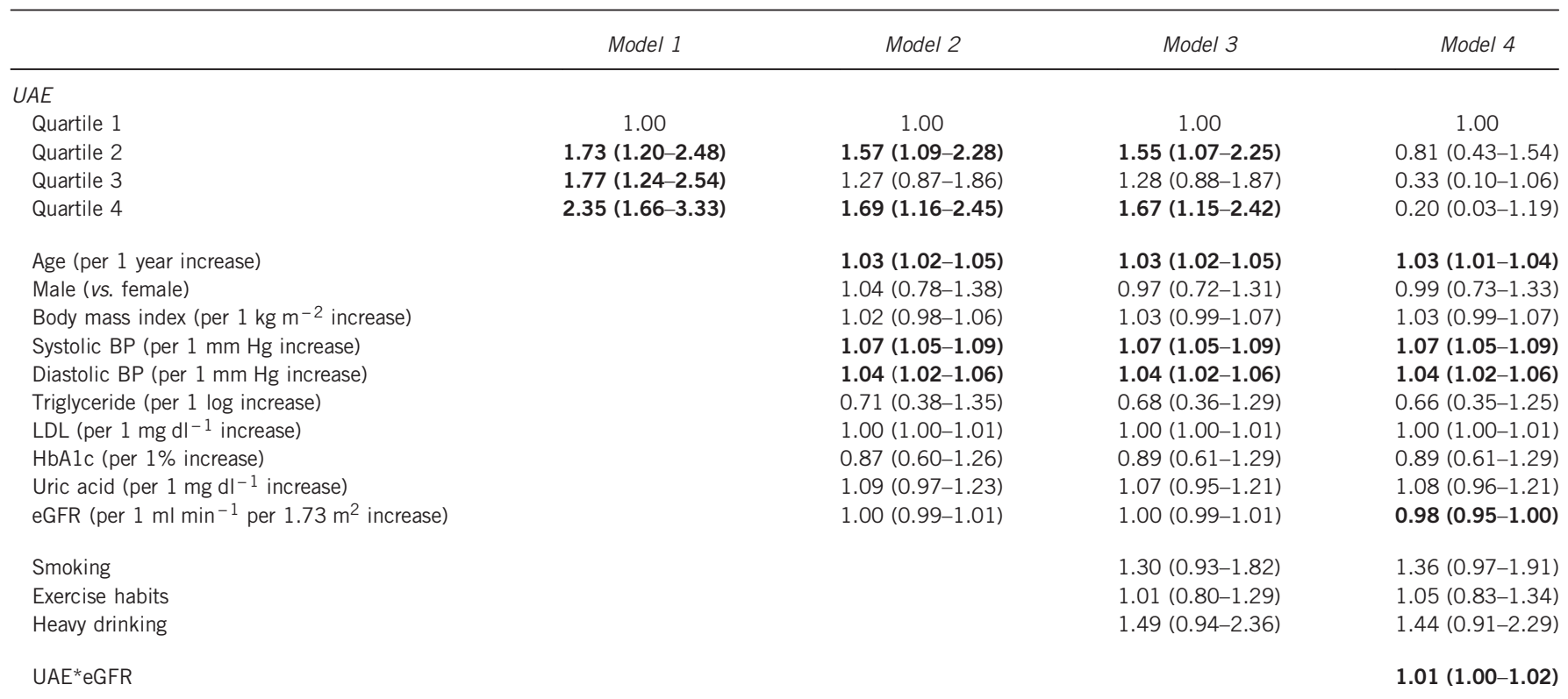

Abbreviations: BP, blood pressure; eGFR, estimated glomerular filtration rate; LDL, low density lipoprotein cholesterol; UAE, urinary albumin excretion.

The bold values indicate statistically significant components.

completely negated when the interaction of UAE and eGFR was added (model 4). The interaction of them was significant $(P=0.018)$. The relationship between UAE quartiles and incident hypertension was examined also by means of logistic regression analysis using same covariates (Supplementary Table 2). The results were essentially similar to those of Cox regression analysis.
To explore the interaction between UAE and eGFR in the prediction of hypertension, we examined the incidence of hypertension in UAE quartiles according to baseline eGFR (Figure 2). Quartile ranges for eGFR were 90 to 169 (Q1), 77 to 89 (Q2), 72 to 76 (Q3) and 40 to $71(\mathrm{Q} 4) \mathrm{ml} \mathrm{min}^{-1}$ per $1.73 \mathrm{~m}^{2}$, respectively. Corresponding median values were 95, 81, 74 and $64 \mathrm{ml} \mathrm{min}^{-1}$ per $1.73 \mathrm{~m}^{2}$, 


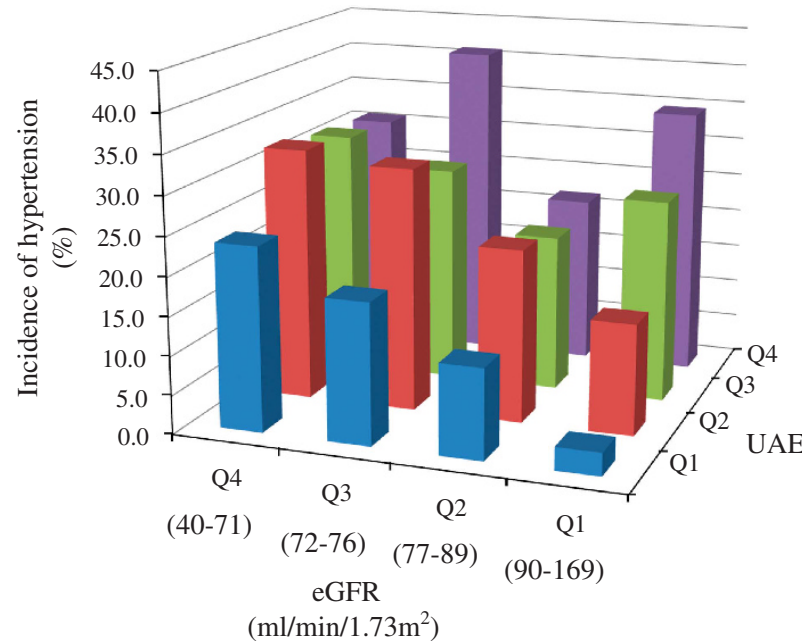

Figure 2 Incidence of hypertension by baseline eGFR (Q1-Q4) and UAE (Q1-Q4).

respectively. The incidence of hypertension was lowest in the first quartile of UAE in the highest GFR group. The incidence of hypertension linearly increased with an increase in baseline UAE in the highest eGFR group $(3.0 \%, 14.3 \%, 26.1 \%$ and $34.6 \%$, respectively, $P<0.001)$. In the first and second UAE quartiles, the lower the eGFR, the higher the incidence of hypertension $(3.0 \%, 11.7 \%, 18.2 \%$ and $23.7 \%, P<0.001$ and $14.3 \%, 22.2 \%, 31.2 \%$ and $32.5 \%, P<0.002$, respectively). A dose-dependent increase in the incidence of hypertension according to UAE categories was absent in the second and forth eGFR quartiles, although the incidence tended to increase with an increase in baseline UAE in the third eGFR quartile $(18.2 \%, 31.2 \%$, $27.9 \%$ and $41.0 \%$, respectively, $P=0.01$ ).

\section{DISCUSSION}

We found the following in the Japanese normotensive general population: (1) subtle increase in UAE was a risk factor for incident hypertension and (2) there was significant interaction between UAE and renal function in the prediction of incident hypertension. The incidence of hypertension dose-dependently increased with an increase in UAE in the highest eGFR quartile over $90 \mathrm{ml} \mathrm{min}^{-1}$ per $1.73 \mathrm{~m}^{2}$. Such a dose-dependent additive effect became smaller and less clear in the lower eGFR categories.

The incidence of hypertension increased with a reduction in eGFR even in the first quartiles of UAE, while the additive effects of UAE became smaller with a reduction in eGFR. In other words, mild renal dysfunction seemed to dominate a subtle increase in UAE in the prediction of incident hypertension.

How can we understand the mechanisms of subtle increases in UAE and renal dysfunction seen in the normotensive general population without overt kidney diseases? What is the reasonable explanation of the interaction between the two variables? An animal model of hypertension showed that one of the earliest findings is an impairment of renal sodium excretion at normal perfusion pressure. ${ }^{2}$ It has been shown in DOCA-SALT hypertension rats, a typical model of volume-dependent hypertension, that continuous elevation in glomerular capillary flow and pressure predispose to glomerular injury and hypertension. ${ }^{16}$ As characteristic features of Japanese hypertension are salt sensitivity and increased salt intake, ${ }^{11}$ this hyperfiltration mechanism may explain, in part, the initial cause of UAE. Waist circumference as well as BPs, triglyceride and $\mathrm{HbAlc}$ were increased with an increase in UAE, despite no significant changes in BMI. This strongly suggests that insulin resistance due to central obesity would be most prevalent in the highest UAE quartile. It is well known that hyperinsulinemia is likely to be associated with hyperfiltration. ${ }^{17,18}$ Thus, subtle UAE may be attributable to hyperfiltration due to insulin resistance especially in the 4th quartile of UAE. This hypothesis is further supported by the fact that highest eGFR individuals were most commonly observed in the 4th quartile of UAE. Moreover, unrecognized diabetes might have contributed to subtle UAE and hyperfiltration in the fourth quartile of UAE. In this study, we excluded subjects with $\mathrm{HbAlc}$ of $6.5 \%$ or over.

However, this criterion is not enough to completely exclude diabetic subjects and therefore we cannot exclude the possibility that considerable number of diabetic subjects were included in the fourth quartile of UAE.

In a spontaneously hypertensive rat model, the degree of renal arteriolar narrowing predicted the extent of future hypertension. ${ }^{19}$ It has also been shown in human that retinal arteriolar narrowing is associated with decline in renal microcirculatory function as captured by eGFR. ${ }^{20}$ Moreover, autopsy studies demonstrated that nephron number was significantly reduced in accident victims with essential hypertension than in matched control individuals. ${ }^{10}$ Reduced nephron number should be associated with decreased filtration capacity and a requirement for higher BP to excrete sodium. ${ }^{21}$ This, on the one hand, could elevate GFRs in the remaining nephrons followed by a chain reaction of increased glomerular pressure and albuminuria. ${ }^{22}$ Taken together, it is plausible that combinations of various degrees of decline in renal filtration capacity, whether congenital or acquired, and endothelial damage could cooperatively initiate high BP.

There have been seven reports examining if the normal range UAE could be a risk for future hypertension in the general population. ${ }^{3-9}$ Among them, only two reported the interaction between UAE and renal function. ${ }^{4,9}$ It has been demonstrated that an increase in UAE synergistically increased the risk of incident hypertension with a lower eGFR in the PREVEND study, ${ }^{4}$ while in the Multi-Ethnic Study of Atherosclerosis, there was no interaction between them. ${ }^{9}$ In MultiEthnic Study of Atherosclerosis, renal function examined by cystatin C was an independent predictor for incident hypertension while normal range UAE was not. Thus, the results of our study are new as compared to these two reports.

The reasons for the difference between our analysis and these previous reports are unclear, but there are several potential explanations. The mean age of our population was 58 years, which is equal to the Multi-Ethnic Study of Atherosclerosis population (58 years) but much higher than that of the PREVEND study (45 years). The mean BMI of this Japanese population is $22.5 \mathrm{~kg} \mathrm{~m}^{-2}$, which is much lower than those of PREVEND or Multi-Ethnic Study of Atherosclerosis. Moreover, diabetes was not excluded from the baseline population in those two studies, in contrast to ours. In the PREVEND study, synergic effects were especially prominent in the microalbuminuria group, which was excluded in our study. These differences in population characteristics could explain the different outcomes.

Our research has an important clinical implication. The prevention of hypertension is an important social issue globally, but the etiology of the development of hypertension largely depends on genetics and environmental factors, even among similar ethnic groups. ${ }^{23}$ To effectively prevent hypertension, we need a proper tool to identify individuals who are at high risk for incident hypertension. Our data showed that assessment of UAE in combination with eGFR, but not UAE alone, can accurately stratify the risk of incident hypertension in the normotensive, non-diabetic Japanese population.

Two similar studies have been performed in East Asian populations, the first in Korea $^{7}$ and the other in Japan. ${ }^{8}$ The former included only a 
select male population who participated in medical check-ups at the university hospital, but the study did not examine the role of the GFR. The latter study included only 412 participants and did not clearly disclose the precise relationship between a subtle increase in UAE and eGFR. We therefore demonstrated for the first time in the Asian general population that a subtle increase in UAE within the normal range could be a risk factor for incident hypertension, dependent upon changes in the GFR.

There are several limitations to this study. First, UAE was examined only once using spot urine samples. We have recently shown, however, that UAE measured in this manner successfully predicted cardiovascular events in this population, ${ }^{14}$ suggesting this is a reliable method. In a previous study, UAE measured through a spot urine sample correlated with measurements from $24 \mathrm{~h}$ collections and national guidelines recommend its use in daily clinical practice. ${ }^{24-26}$ Second, we used plasma creatinine to calculate GFR, which might reduce the accuracy for detection of early kidney dysfunction compared with cystatin C. ${ }^{27,28}$ In other words, use of cystatin $\mathrm{C}$ may more precisely examine the interaction between renal function and UAE in the prediction of incident hypertension. Third, we did not examine the information on salt intake, which must be potentially important as a pathogenic factor in Japanese hypertension. Finally, we did not study the relationship with longitudinal changes in BP and those in UAE or GFR. Such analysis may disclose complex relationship between BP progression and renal physiology and clarify more the role of kidney for incident hypertension. Some of the limitations of this study will be considered in the future.

In conclusion, we showed that a subtle increase in UAE within the normal range could be a risk factor for incident hypertension. The risk of incident hypertension dose-dependently increased in the highest eGFR group. Decline in renal function alone increased the risk of incident hypertension, but the additional risk with a subtle increase in UAE became smaller and less clear in the lower eGFR categories. Therefore, $\mathrm{UAE}$ as a risk factor for incident hypertension was largely dependent on eGFR levels. Our data suggest that the hypertension is initiated by the combination of various degree of decline in renal filtration capacity and endothelial damage in the Japanese general population.

\section{CONFLICT OF INTEREST}

The authors declare no conflict of interest.

\section{ACKNOWLEDGEMENTS}

This study was supported by the grants and aid from the Japan Organization of Occupational Health and Safety.

1 Lawes CM, Vander Hoorn S, Rodgers, Alnternational Society of Hypertension. Global burden of blood-pressure-related disease, 2001. Lancet 2008; 371: 1513-1518.

2 Cowley AW Jr., Roman RJ. The role of the kidney in hypertension. JAMA 1996; 275: 1581-1589.

3 Wang TJ, Evans JC, Meigs JB, Rifai N, Fox CS, D'Agostino RB, Levy D, Vasan RS. Lowgrade albuminuria and the risks of hypertension and blood pressure progression. Circulation 2005; 111: 1370-1376.

4 Brantsma AH, Bakker SJ, de Zeeuw D, de Jong PE, Gansevoort RT. Urinary albumin excretion as a predictor of the development of hypertension in the general population. $J$ Am Soc Nephrol 2016; 17: 331-335.

5 Forman JP, Fisher ND, Schopick EL, Curhan GC. Higher levels of albuminuria within the normal range predict incident hypertension. J Am Soc Nephrol 2008; 19: 1983-1988.

6 Jessani S, Levey AS, Chaturvedi N, Jafar TH. High normal levels of albuminuria and risk of hypertension in Indo-Asian population. Nephrol Dial Transplant 2012; 27 (Suppl 3): iii58-iii64.

7 Park SK, Moon SY, Oh CM, Ryoo JH, Park MS. High normal urine albumin-to-creatinine ratio predicts development of hypertension in Korean men. Circ J 2014; 78: 656-661.
8 Hirayama A, Konta T, Hozawa A, Kawasaki R, Watanabe T, Shibata Y, Kayama T, Fukao A, Kubota I. Slight increase in urinary albumin excretion within the normal range predicts incident hypertension in a community-based Japanese population: the Takahata study. Hypertens Res 2015; 38: 56-60.

9 Kestenbaum B, Rudser KD, de Boer IH, Peralta CA, Fried LF, Shlipak MG, Palmas W, Stehman-Breen C, Siscovick DS. Differences in kidney function and incident hypertension: the multi-ethnic study of atherosclerosis. Ann Intern Med 2008; 148: 501-508.

10 Keller G, Zimmer G, Mall G, Ritz E, Amann K. Nephron number in patients with primary hypertension. N Engl J Med 2003; 9348: 101-208.

11 Kokubo Y. Prevention of hypertension and cardiovascular diseases: a comparison of lifestyle factors in Westerners and East Asians. Hypertension 2014; 63: 655-660.

12 Munakata M, Konno S, Ohshima M, Ikeda T, Miura Y, Ito S. High-normal blood pressure is associated with microalbuminuria in the general population: the Watari study. Hypertens Res 2011; 34: 1135-1140.

13 Konno S, Hozawa A, Miura Y, Ito S, Munakata M. High-normal diastolic blood pressure is a risk for development of microalbuminuria in the general population: the Watari study. J Hypertens 2013; 31: 798-804.

14 Konno S, Munakata M. Moderately increased albuminuria is an independent risk factor of cardiovascular events in the general Japanese population under 75 years of age: the Watari study. PLOS ONE 2015; 10: e0123893.

15 Hattori T, Konno S, Munakata M. Gender differences in lifestyle factors associated with metabolic syndrome and preliminary metabolic syndrome in the general population: the Watari study. Intren Med 2017; 56: 2253-2259.

16 Dworkin LD, Hostetter TH, Rennke HG, Brenner BM. Hemodynamic basis for glomerular injury in rats with desoxycorticosterone-salt hypertension. J Clin Invest 1984; 73: 1448-1461.

17 Tomaszewski M, Charchar FJ, Maric C, McClure J, Crawford L, Grzeszczak W, Sattar N, Zukowska-Szczechowska E, Dominiczak AF. Glomerular hyperfiltration: a new marker of metabolic risk. Kidney Int 2007; 71: 816-821.

18 Naderpoor N, Lyons JG, Mousa A, Ranasinha S, Courten MP, Soldatos G, Courten B. Higher glomerular filtration rate is related to insulin resistance but not to obesity in a predominantly obese non-diabetic cohort. Sci Rep 2017; 3745522.

19 Nørrelund H, Christensen KL, Samani NJ, Kimber P, Mulvany MJ, Korsgaard N. Early narrowed afferent arteriole is a contributor to the development of hypertension. Hypertension 1994; 24: 301-308.

20 Gu YM, Petit T, Wei FF, Thijs L, Jacobs L, Zhang ZY, Yang WY, Cauwenberghs N, Knez J, Struijker-Boudier HA, Kuznetsova T, Verhamme P, Staessen JA. Renal glomerular dysfunction in relation to retinal arteriolar narrowing and high pulse pressure in seniors. Hypertens Res 2016; 39: 138-143.

21 Kimura G, Brenner B. In Laragh JH, Brenner BM (eds). Hypertension: Pathophysiology, Diagnosis, and Management, 2nd edn. Raven Press, 1005: 1569-1588.

22 Brenner BM, Garcia DL, Anderson S. Glomeruli and blood pressure. Less of one, more the other? Am J Hypertens 1988; 1: 335-347.

23 Seedat YK. Race, environment and blood pressure: the South African experience. $J$ Hypetens 1983; 1: 7-12.

24 Nathan DM, Rosenbaum C, Protasowicki VD. Single-void urine samples can be used to estimate quantitative microalbuminuria. Diabetes Care 1987; 10: 414-418.

25 Bakker AJ. Detection of microalbuminuria. Receiver operating characteristic curve analysis favors albumin-to-creatinine ratio over albumin concentration. Diabetes Care 1999; 22: 307-313.

26 Levey AS, Coresh J, Balk E, Kausz AT, Levin A, Steffes MW, Hogg RJ, Perrone RD, Lau J, Eknoyan G; National Kidney Foundation. National Kidney Foundation practice guidelines for chronic kidney disease: evaluation, classification, and stratification. Ann Intern Med 2003; 139: 137-147.

27 Coll E, Botey A, Alvarez L, Poch E, Quintó L, Saurina A, Vera M, Piera C, Darnell A. Serum cystatin $C$ as a new marker for noninvasive estimation of glomerular filtration rate and as a marker for early renal impairment. Am J Kidney Dis 2000; 36: 29-34.

28 Newman DJ, Thakkar H, Edwards RG, Wilkie M, White T, Grubb AO, Price CP. Serum cystatin $\mathrm{C}$ measured by automated immunoassay: a more sensitive marker of changes in GFR than serum creatinine. Kidney Int 1995; 47: 312-318.

(c) (i) (-) $\Theta$ This work is licensed under a Creative Commons Attribution-NonCommercial-NoDerivs $\quad 4.0$ International License. The images or other third party material in this article are included in the article's Creative Commons license, unless indicated otherwise in the credit line; if the material is not included under the Creative Commons license, users will need to obtain permission from the license holder to reproduce the material. To view a copy of this license, visit http://creativecommons.org/licenses/bync-nd/4.0/

(C) The Author(s) 2017

Supplementary Information accompanies the paper on Hypertension Research website (http://www.nature.com/hr) 